\title{
Vaccinia virus-mediated intra-tumoral expression of matrix metalloproteinase 9 enhances oncolysis of PC-3 xenograft tumors
}

\author{
Simon Schäfer ${ }^{1}$, Stephanie Weibel ${ }^{1}$, Ulrike Donat ${ }^{1}$, Qian Zhang ${ }^{2,3}$, Richard J Aguilar ${ }^{2}$, Nanhai G Chen ${ }^{2,3}$ \\ and Aladar A Szalay ${ }^{1,2,3^{*}}$
}

\begin{abstract}
Background: Oncolytic viruses, including vaccinia virus (VACV), are a promising alternative to classical mono-cancer treatment methods such as surgery, chemo- or radiotherapy. However, combined therapeutic modalities may be more effective than mono-therapies. In this study, we enhanced the effectiveness of oncolytic virotherapy by matrix metalloproteinase (MMP-9)-mediated degradation of proteins of the tumoral extracellular matrix (ECM), leading to increased viral distribution within the tumors.

Methods: For this study, the oncolytic vaccinia virus GLV-1h255, containing the mmp-9 gene, was constructed and used to treat PC-3 tumor-bearing mice, achieving an intra-tumoral over-expression of MMP-9. The intra-tumoral MMP-9 content was quantified by immunohistochemistry in tumor sections. Therapeutic efficacy of GLV-1h255 was evaluated by monitoring tumor growth kinetics and intra-tumoral virus titers. Microenvironmental changes mediated by the intra-tumoral MMP-9 over-expression were investigated by microscopic quantification of the collagen IV content, the blood vessel density (BVD) and the analysis of lymph node metastasis formation.

Results: GLV-1h255-treatment of PC-3 tumors led to a significant over-expression of intra-tumoral MMP-9, accompanied by a marked decrease in collagen IV content in infected tumor areas, when compared to GLV-1h68-infected tumor areas. This led to considerably elevated virus titers in GLV-1h255 infected tumors, and to enhanced tumor regression. The analysis of the BVD, as well as the lumbar and renal lymph node volumes, revealed lower BVD and significantly smaller lymph nodes in both GLV-1h68- and GLV-1h255- injected mice compared to those injected with PBS, indicating that MMP-9 over-expression does not alter the metastasis-reducing effect of oncolytic VACV.

Conclusions: Taken together, these results indicate that a GLV-1h255-mediated intra-tumoral over-expression of MMP-9 leads to a degradation of collagen IV, facilitating intra-tumoral viral dissemination, and resulting in accelerated tumor regression. We propose that approaches which enhance the oncolytic effect by increasing the intra-tumoral viral load, may be an effective way to improve therapeutic outcome.
\end{abstract}

\section{Background}

Prostate cancer $(\mathrm{PCa})$ is responsible for $11 \%$ of cancerrelated deaths in men in the US, second only to lung and bronchial cancer cases [1]. In most patients, death is caused by the formation of metastases. Therefore, the success of therapy is strikingly dependent on the time point

\footnotetext{
* Correspondence: aaszalay@genelux.com

'Department of Biochemistry, Biocenter, University of Würzburg, Würzburg 97074, Germany

${ }^{2}$ Genelux Corporation, San Diego Science Center, San Diego, CA 92109, USA Full list of author information is available at the end of the article
}

of diagnosis [2], and the efficacy of the therapy. Commonly used classical cancer therapies still show deficits in regard to their efficacy and/ or specificity. An exemplary obstacle for successful treatment is the development of resistance to chemo- or radiotherapy [3,4]. In the case of surgery, malignant cells can remain after the primary tumor is removed, which might lead to enhanced overall survival, but not a complete recovery [5].

Therefore, it is necessary to investigate alternative treatments. A promising therapeutic approach for the treatment of $\mathrm{PCa}$ are oncolytic viruses such as vaccinia

\section{Ciomed Central}


virus (VACV) GLV-1h68 [6]. This virus was also successfully used in other malignancies such as pancreatic tumors, squamous cell carcinoma, breast cancer or in combination with a prodrug activated by the GLV-1h68carried marker gene $\beta$-galactosidase [7-10]. VACV replicates exclusively in the cytoplasm [11] and has a large genome well suited for inserts of up to $25 \mathrm{~kb}$ [12]. This fact was exploited for the construction of further recombinant VACVs (rVACVs). The inserted genes can be used to enhance therapeutic efficacy as in the case of a GLAF-1 (a single chain antibody against vascular endothelial growth factor (VEGF)) encoding VACV [13], or to allow the use of imaging techniques such as positron emission tomography (PET) [14,15].

In this study, the possibility to use oncolytic viruses as a vector for the intra-tumoral expression of recombinant proteins was explored to further enhance virotherapy by elevating intra-tumoral titers, which may increase the oncolytic effect. Generally, the extracellular matrix (ECM) can be a hindrance for viral cell-to-cell spreading, as reported for oncolytic adenoviruses, decreasing the oncolytic effect. However, it was shown that the insertion of the relaxin gene in adenoviruses leads to the degradation of ECM components within the tumor microenvironment, thus improving viral spreading $[16,17]$. Further possible candidates, which can be used for the intra-tumoral degradation of ECM components, are the matrix-metalloproteinases (MMPs) [18]. MMPs are zinc-dependent endopeptidases, usually expressed as inactive zymogens. In their activated state, these MMPs are important for the breakdown of several ECM proteins, playing a role in various processes (e.g. tissue remodeling) and also in cancer progression $[19,20]$. In addition, the cleavage of ECM proteins can result in the shedding of ECM-bound growth factors, leading to multiple actions within the tumor microenvironment $[21,22]$. For example, not only has an enhanced angiogenesis been observed after MMP-mediated shedding of pro-angiogenic factors (e.g. VEGF), but also a decreased BVD due to anti-angiogenic factors (e.g. endostatin, tumstatin, angiostatin) was reported [23-25].

In this study, the oncolytic VACV GLV-1h255, containing the $m m p-9$ gene, was constructed to specifically degrade the intra-tumoral ECM. Among the MMP-9 ECM substrates are collagen, laminin, fibrillin and elastin [26]. Indeed, we could observe a significantly decreased collagen IV content, accompanied by increased intra-tumoral virus titers in GLV-1h255colonized PC-3 tumors. Additionally, the tumor regression process started earlier in the treatment phase and was markedly accelerated. Our results suggest that a virus-mediated intra-tumoral degradation of ECM components offers a new strategy to optimize oncolytic rVACV tumor treatment.

\section{Methods}

\section{Cell culture}

PC-3 (DSMZ no. ACC465) and A549 (ATCC no. CCL185) cells were cultured in RPMI 1640 supplemented with $10 \%$ fetal bovine serum (FBS) and antibiotic solution (100 units $/ \mathrm{mL}$ penicillin $\mathrm{G}$ and 100 units $/ \mathrm{mL}$ streptomycin) at $37^{\circ} \mathrm{C}$ and $5 \% \mathrm{CO}_{2}$.

For CV-1 cells (provided by Genelux GmbH, Bernried) DMEM instead of RPMI 1640 was used. Media and additives were purchased from PAA (Pasching, Austria).

\section{Construction of recombinant VACV GLV-1h255}

The human MMP-9 cDNA clone was originally from OriGene (Cat. SC116989), which encodes the Homo sapiens matrix metallopeptidase 9 (Accession No. NM_004994.2). The human MMP-9 cDNA was PCR amplified with primers MMP9-5 [5'-GTCGAC(Sal I) CACCATGAGCCTCTGGCAGCCC-3'] and MMP9-3 [5'-TTAATTAA(PacI) CTAGTCCTCAGGGCACTGCA$\left.3^{\prime}\right]$ with the above mentioned clone as the template. The PCR product was gel purified, and cloned into the pCRBlunt II-TOPO vector using the Zero Blunt TOPO PCR Cloning Kit (Invitrogen). The resulting construct pCRIIMMP9-1 was sequence confirmed. The MMP9 cDNA was released from pCRII-MMP9-1 with Sal I and Pac I, and subcloned into the TK transfer vector with the same restriction sites, placing hMMP9 cDNA under the control of vaccinia early (SE) promotor. The resulting construct TK-SE-hMMP9 was sequence confirmed.

TK-SE-hMMP9 was used to generate the recombinant virus GLV-1h255, with GLV-1h68 as the parental virus, replacing the p7.5-lacZ expression cassette and pSELhTFR in the TK locus with the expression cassette of hMMP-9.

\section{MMP-9 protein analysis}

PC-3 cells were infected with GLV-1h68 or GLV-1h255 at a multiplicity of infection (MOI) of 1. Supernatants were collected $24 \mathrm{~h}$ post infection (p.i.) and sterile-filtred (0.1 $\mu \mathrm{m}$ filter Schleicher \& Schuell Dassel, Germany). Cells were washed once with PBS and lysed by addition of SDS sample buffer $(130 \mathrm{mM}$ Tris $\mathrm{HCl} \mathrm{pH} \mathrm{6.8,} \mathrm{4 \%} \mathrm{(w/v)}$ SDS, 20\% (v/v) glycerol, 5.3\% (v/v) 2-mercaptoethanol, $0.15 \mathrm{mM}$ bromphenol blue). Supernatants were mixed (1:1) with SDS sample buffer. All samples were denatured, separated by SDS-PAGE and transferred to nitrocellulose membranes. MMP-9 was detected using a polyclonal antiMMP-9 antibody produced in goat (Neuromics, Edina USA, GT15020) and a HRP-conjugated anti-goat antibody (Sigma, Steinheim Germany, A8919). ECL solution (90 mM p-coumaric acid, $250 \mathrm{mM}$ luminol, $1 \mathrm{M}$ Tris$\mathrm{HCl}, \mathrm{pH}$ 8.5) was used as a substrate for the HRP reaction. $\beta$-actin was detected using an anti- $\beta$-actin antibody produced in mouse (Abcam, Cambridge UK, ab6276) and a 
HRP-conjugated anti-mouse antibody (Abcam, Cambridge UK, ab6728).

For the zymography (Millipore, protocol MCPROTO 009, 2007), A549 cells were infected with GLV-1h68 or GLV-1h255 at an MOI 0.1 for $24 \mathrm{~h}$. At this time point supernatants were collected and sterile-filtered. Cells were washed once with PBS and lysed by addition of non-reducing SDS sample buffer (w/o $\beta$-mercaptoethanol). Supernatants were mixed (1:1) with non-reducing SDS sample buffer. Protein samples were separated by SDS-PAGE using 10\% SDS gels containing $1 \mathrm{mg} / \mathrm{mL}$ gelatin. After separation the proteins were renatured and developed for $1 \mathrm{~d}$ at $37^{\circ} \mathrm{C}$. Gel staining was performed with $0.5 \%(\mathrm{w} / \mathrm{v})$ Coomassie Brilliant Blue.

\section{Animal studies}

PC-3 cells $\left(2 \times 10^{6}\right.$ in $100 \mu \mathrm{L}$ PBS $)$ were implanted subcutaneously in the right abdominal flank of $6-8$ week old female nude mice (NCl:Hsd:Athymic Nude Foxn $1^{n u}$, Harlan Borchem, Germany). Tumors were measured weekly in two dimensions using a digital caliper and tumor volumes were calculated (length $\times$ width $^{2} / 2$ ). After approximately 3 weeks, a single dose of GLV-1h68 or GLV-1h255 $\left(5 \times 10^{6}\right.$ plaque forming units (pfu) in 100 $\mu \mathrm{L}$ PBS) was injected into the tail vein (i.v.). Control animals were injected with $100 \mu \mathrm{L}$ PBS. All animal experiments were carried out in accordance with protocols approved by the Regierung of Unterfranken (Würzburg, Germany, protocol number AZ 55.2-2531.01-17/08) and/ or the Institutional Animal Care and Use Committee (IACUC) of Explora BIOLABS, located in the San Diego Science Center (San Diego, USA) (protocol number: EB08-003).

\section{Viral titration in tumor samples}

Tumors were snap-frozen in liquid nitrogen. After thawing tumors were weighed, cut into pieces using a scalpel and transferred to M-tubes (Miltenyi Biotec, Bergisch Gladbach, Germany) and mixed with the $2 \times$ volume of buffer (1 tablet of Roche complete mini proteinase inhibitor dissolved in $50 \mathrm{~mL}$ of PBS). Homogenization of tissues was done twice with a GentleMACS dissociator (Miltenyi Biotec). Subsequently, samples underwent 3 freeze/thaw cycles and were sonified 3 times for $30 \mathrm{~s}$ each time. Virus titers were obtained by performing a standard plaque assay on $\mathrm{CV}-1$ cells.

\section{Virus replication assay}

To analyze the in vitro growth kinetics of GLV-1h68 and GLV-1h255, PC-3 cells were seeded in 24 well plates. When the cell layer reached 90\% confluency, cells were infected with an MOI of 0.1 in triplicates for each time point. At 1, 2, 4, 6, 10, 24, 48 and 72 hours post infection supernatants were obtained and cells were washed with Hank's BSS (PAA, Pasching, Austria). Cells were detached using trypsin and pelleted by centrifugation and resuspended in Hank's BSS. All samples were snap frozen in liquid nitrogen and stored at $-80^{\circ} \mathrm{C}$. To obtain the viral titers, a standard plaque assay on $\mathrm{CV}-1$ cells was performed. Prior to the plaque assay, three freezeand-thaw cycles were completed to achieve a thorough cell lysis.

\section{Analysis of lumbar and renal lymph nodes}

For the analysis of lymph node metastases in PC-3 tumor bearing mice, mice were sacrificed 24 days after virus treatment as described above. To calculate the volumes of lumbar and renal lymph node metastases, the abdomen was opened and all organs were removed. A picture of the now visible lymph nodes was taken, using a ruler as a guide. The width and length were measured using Photoshop CS4 (Adobe Systems, Mountain View, CA). Lymph node volumes were calculated using the formula: length $\times$ width $^{2} / 2$.

\section{Histology, fluorescence microscopy and image analysis}

$100 \mu \mathrm{m}$ tumor sections were prepared as described previously [27]. All of the following steps were carried out at room temperature. Tissue sections were permeabilized by incubation in PBS containing $0.2 \%$ Triton X-100 and $5 \%$ FBS (blocking solution) for $1 \mathrm{~h}$. For fluorescence labeling, sections were incubated for $12 \mathrm{~h}$ with the primary antibody in blocking solution. Subsequently, sections were washed with PBS and incubated with the corresponding secondary antibody in blocking solution for $4 \mathrm{~h}$. Finally, tumor sections were washed in PBS and mounted onto glass slides using Mowiol/DABCO.

The fluorescence labeled specimens were examined using a stereo-fluorescence microscope (MZ16 FA, Leica) equipped with a color CCD camera (DC500, Leica). Images were acquired using the Leica IM1000 v 4.0 software and Photoshop CS4 was employed to adjust levels and create overlays.

Collagen IV in PC-3 tumor sections was labeled with an anti-collagen IV antibody (Abcam, Cambridge UK, ab19808). Quantification was performed using ImageJ (http://rsbweb.nih.gov/ij/). Three images (100x, Leica MZ16 FA) from infected and three images from noninfected areas were taken from each section. Images taken from 3 non-consecutive sections for each tumor, and 3 tumors per group were used for analysis.

MMP-9 expression was analyzed in PC-3 tumor sections labeled with MMP-9 antibody (Neuromics, Edina USA, GT15020). Image acquisition was done as described before, except that a magnification of $150 \mathrm{x}$ was used (Leica MZ16 FA). Visualization of MMP-9 expression was also done with these sections. All images were converted from RGB to grayscale for ImageJ 
analysis (Photoshop CS4), with mean fluorescence intensity measurements reported.

\section{Analysis of blood vessel density}

The blood vessel density was determined using microscopic images (200x, Leica MZ16 FA) of CD31-labeled (CD31 antibody, Millipore, mab1398Z) tumor sections. Three non-consecutive sections from each tumor were taken and 3 tumors per group were analyzed. For each section, 3 images from infected and 3 from noninfected areas were taken. Exposure time was adjusted to ensure clear visibility of the blood vessels in each section. Subsequently, 5 equidistant lines were drawn in a template using Adobe Illustrator CS4. Each individual image was superimposed with the template and vessels crossing the lines were counted. The average blood vessel density per section with standard deviation is shown.

\section{Statistical analysis}

A two-tailed Student's $t$ test was used to determine statistical significance ( $p$ values: $* p<0.05$, * $p<0.01$, **** $p<0.001)$. Error bars represent standard deviation.

\section{Results}

MMP-9 is expressed in GLV-1h255 infected PC-3-cells and shows enzymatic activity in vitro

In this study, the rVACV GLV-1h255 was constructed to express human MMP-9 under control of the synthetic early promoter as shown in (Figure 1A). The expression of MMP-9 by GLV-1h255 infected PC-3 cells was confirmed by Western Blot (Figure 1B). To investigate enzymatic activity of secreted MMP-9 by zymography, we infected A549 lung carcinoma cells with either GLV1 h68 or GLV-1h255. In A549 cells, which are well suited for protein over-expression, abundant protein expression allowed the visualization of enzymatic activity of MMP-9 in both cell-lysates and the sterile-filtered supernatants (Figure 1C).

\section{MMP-9 is expressed in GLV-1h255 infected areas of PC-3 tumors}

Expression of MMP-9 was also analyzed in PC-3 tumor sections at 7 days p.i.. In PC-3 tumors from either PBS or GLV-1h68 treated animals, an already high basal expression level of MMP-9 was detected, probably associated with immune cells. Nonetheless, a specific MMP-9 over-

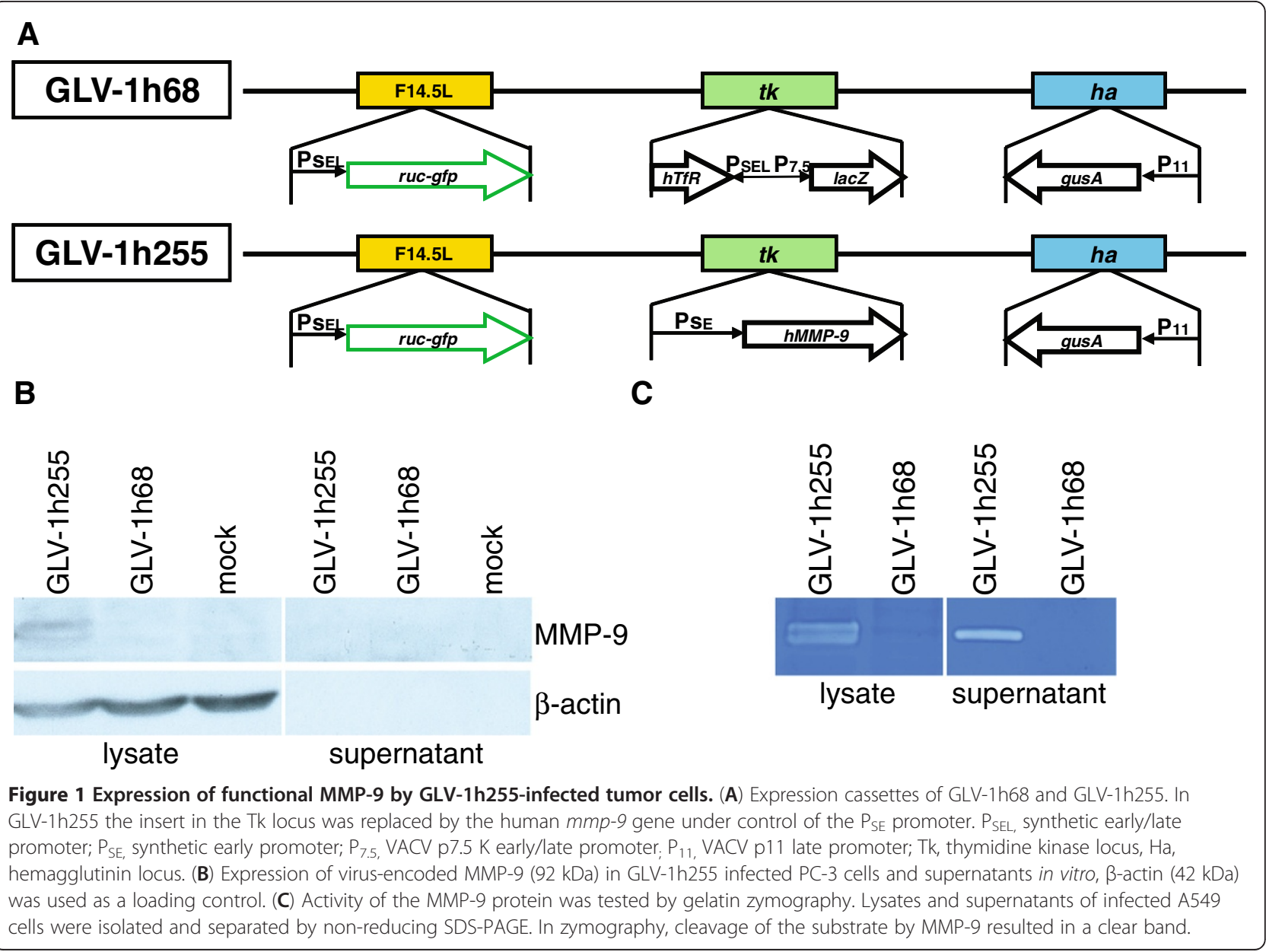


expression was observed in GLV-1h255 infected tumors, compared to tumors of both control groups (Figure 2A). The over-expression of MMP-9 co-localized with GFPpositive GLV-1h255-infected tumor areas. In addition, the MMP-9 expression was significantly higher in GLV-1h255 infected areas compared to non-infected areas (Figure 2B). In comparison, MMP-9 expression in GLV-1h68 infected areas was considerably lower than in GLV-1h255 infected areas.

\section{Virus-mediated MMP-9 expression enhances collagen IV} degradation in GLV-1h255 infected tumor areas

To analyze whether MMP-9 activity in GLV-1h255 infected tumors leads to the degradation of ECM components, we investigated the distribution of collagen IV, a protein of the ECM and substrate of MMP-9, in tumors. Indeed, in infected areas of GLV-1h255 infected tumors, the collagen IV content was significantly lower than in GLV-1h68 infected areas (Figure 2C). In general, however, we observed a significantly higher collagen IV content in GLV-1h68 infected tumors in comparison to PBS treated tumors. This increase might be due to the viral infection, leading to an uncontrolled inflammation in the tumor microenvironment (reviewed in [28]), which may result in a pronounced fibrotic deposition of collagen. Therefore, virus-mediated MMP-9 expression might minimize infection-related collagen IV fibrosis.

\section{GLV-1h255 treatment of tumors accelerates the tumor} regression process by increasing intra-tumoral virus titers To determine whether the virus-mediated expression of MMP-9 and degradation of collagen IV may improve

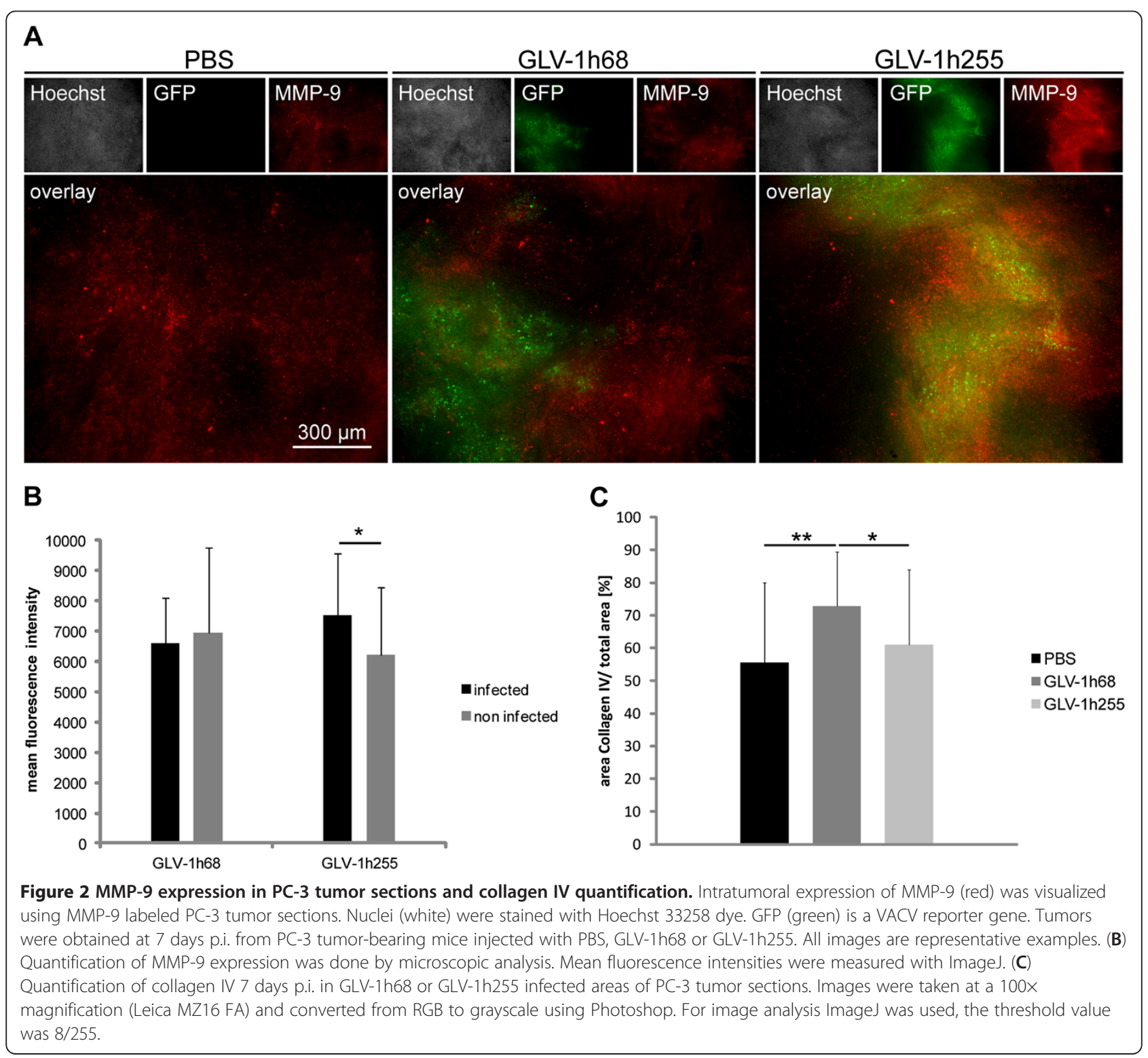


oncolytic viral therapy, PC-3 tumor-bearing mice were injected with a single dose of GLV-1h255. Control animals were injected with either GLV-1h68 or PBS. The three phased growth pattern (growth, inhibition, regression) of PC-3 xenograft tumors after viral injection was in accordance with previous observations [10]. Significant tumor regression already occurred at 17 days p.i. in GLV-1h255 treated animals, but not in those treated with GLV-1h68. This indicates an enhanced oncolytic effect of GLV-1h255, compared to the parental GLV-1h68 virus. Furthermore, tumors of GLV-1h255 injected animals showed a significantly enhanced regression at 24 days p.i. when compared with PBS injected mice $(p<0.001)$, whereas the difference in volumes between GLV-1h68 and PBS treated tumors was also significant, but to a lesser extend $(p<0.01)$ (Figure 3A).

Since the primary idea for the construction of GLV1h255 was to increase viral spreading and viral load in infected tumors, enhancing the oncolytic effect, we analyzed virus titers in GLV-1h68 and GLV-1h255 infected tumors at 7 days p.i. by plaque assay. Indeed, at this early time point after infection, GLV-1h255 infected tumors showed a significantly higher average virus titer than those infected with GLV-1h68 (Figure 3B). To exclude the possibility that the higher titers were due to a better replication of GLV-1h255, in vitro replication in PC-3 cells was analyzed by a replication assay (Figure 4). In this case the replication of GLV-1h255 was not enhanced when compared to the replication of GLV-1h68.

Over-expression of MMP-9 in GLV-1h255 infected tumors does not lead to an enhanced angiogenesis

Angiogenesis is often linked to elevated expression levels of MMP-9 as it influences the bioavailability of VEGF

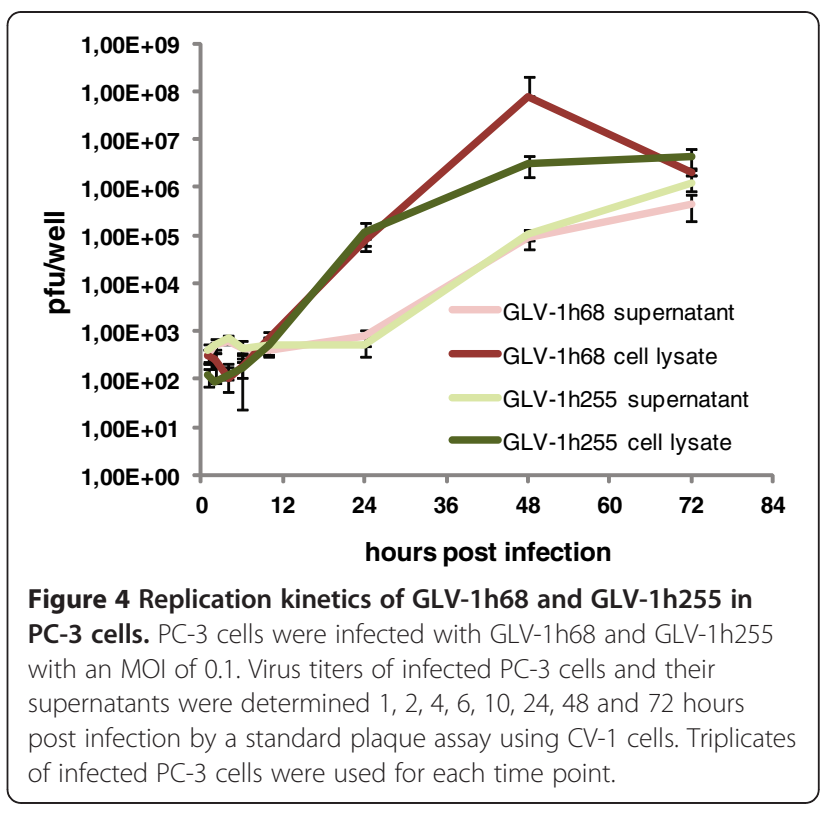

[29]. Therefore, intra-tumoral over-expression of MMP9 could theoretically be associated with enhanced angiogenesis. We determined the vascular density in PC-3 tumor sections at 7 days p.i. to elucidate whether it is influenced by the GLV-1h255 mediated over-expression of MMP-9 (Figure 5A). In GLV-1h68 or GLV-1h255 infected areas of tumors, the vascular density was significantly lower than in uninfected areas or sections from tumors of PBS injected animals. Additionally, there was no significant difference in the BVD between GLV-1h68and GLV-1h255-infected tumor areas. Thus, GLV-1h255 treatment of PC-3 tumor-bearing mice did not enhance angiogenesis.

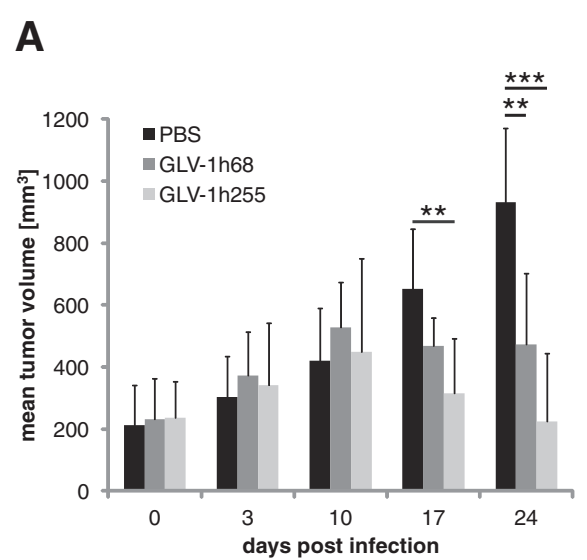

\section{B}

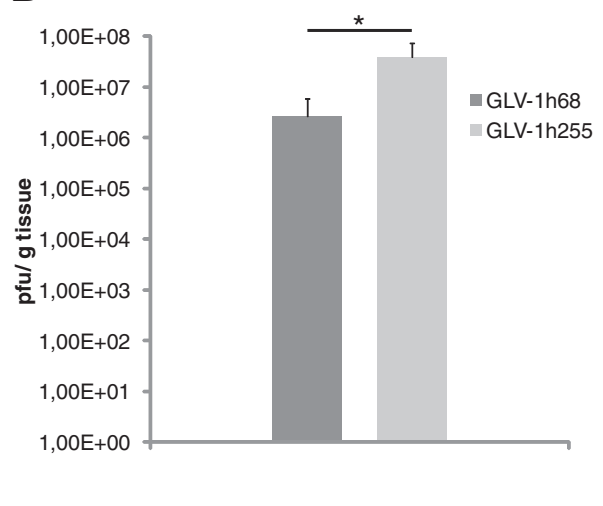

Figure 3 Tumor regression analysis and virus titers in GLV-1h68 and GLV-1h255 infected tumors. (A) Growth of PC-3 xenograft tumors in athymic nude mice. Animals were i.v. injected with GLV-1h68, GLV-1h255 or PBS. Tumor volumes were measured weekly $(n=6-7)$. Statistical significance was determined by using a two-tailed Student's $t$ test ( $p$-values: ${ }^{*}<0.05,{ }^{* *}<0.01,{ }^{* * *}<0.001$ ). (B) Viral titers (pfu/g tissue) of tumor homogenates 7 days p.i.. Titers were obtained by performing a plaque assay on CV-1 cells. Four tumors per group (GLV-1h68, GLV-1h255) were used. 


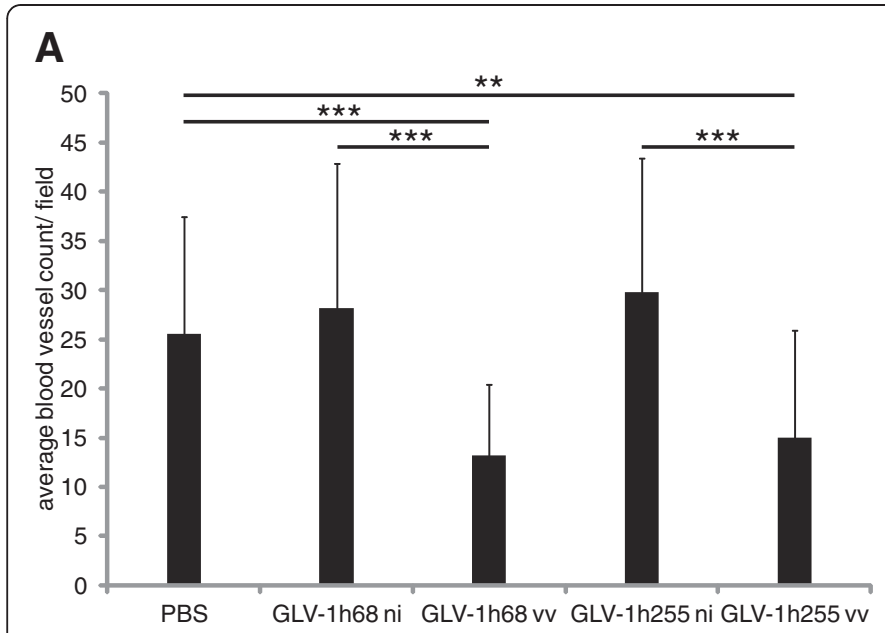

B

Figure 5 Analysis of lumbar and renal lymph node metastases in PC-3 tumor-bearing mice and of Here, the blood vessel density should be mentioned first (Figure 5A, text labeled in yellow) and lymph nodes second (Figure 5B). (A) Blood vessel density in PC-3 tumor sections, 7 days p.i.. For GLV-1h68 and GLV-1h255 blood vessels in infected (vV) and non-infected (ni) areas were counted. Infected areas were determined by expression of the viral marker GFP. For each image (200x, Leica MZ16 FA) blood vessels crossing 5 equidistant lines were counted. (B) Analysis of renal and lumbar lymph node enlargement. PC-3 tumor-bearing mice were injected i.v. with GLV-1h68, GLV-1h255 or PBS. The mice were sacrificed 24 days p.i. and lymph nodes were measured $(n=6-7)$.

\section{GLV-1h68 and GLV-1h255 have a strong therapeutic} effect on renal and lumbar lymph node metastases in PC-3 tumor-bearing mice

PC-3 xenograft tumors have a high metastatic potential in athymic nude mice [30-32] and the expression of ECM-degrading enzymes might additionally increase the shedding of tumor cells, resulting in elevated metastasis formation. Of these metastases, the renal and lumbar ones can be easily analyzed after a removal of organs from the abdomen. To determine whether the intratumoral expression of MMP-9 in GLV-1h255 treated PC-3 tumors influence the metastatic spread, the volume of renal and lumbar lymph nodes was measured 24 days p.i. (Figure 5B). The renal and lumbar lymph nodes in GLV-1h255 injected mice had similar volumes as in GLV-1h68 treated mice, and both were significantly smaller than in PBS injected ones. Therefore, the virus-mediated over-expression of MMP-9 enhances virotherapy of the primary tumor while sustaining the rVACV-metastasis reducing effect.

\section{Discussion}

The use of oncolytic VACV is a promising approach for the treatment of various types of cancer [7-10]. However, the therapeutic efficacy relies extensively on the efficient oncolysis of tumor cells [27], indicating that an efficient viral spreading inside tumor mass is essential. In this context, proteins of the ECM have been identified as an obstacle for the intra-tumoral spreading of virus particles $[33,34]$.
Two recently published studies reported that adenovirus-mediated intra-tumoral expression of relaxin resulted in an increased tumor regression [16,17].

In the present study, we showed for the first time that degradation of the tumoral ECM also enhanced therapeutic efficacy of oncolytic rVACV. To achieve tumoral ECM degradation, we used GLV-1h255 encoding $m m p-9$, for the treatment of PC-3 tumor-bearing mice.

PC-3 tumors of GLV-1h68 and PBS injected mice showed a high basal MMP-9 expression. This might be due to the presence of non-malignant stromal cells, e.g. inflammatory cells such as neutrophils, macrophages or lymphocytes, most of which express MMP-9 [22]. Since PC-3 xenograft tumors have a high metastatic potential [30-32] and the degradation of ECM proteins by MMP-9 may facilitate metastasis [21], we analyzed the size of lumbar and renal lymph nodes in PC-3 tumor bearing mice, as these have been shown to be colonized by tumor cells, and increased in size compared to healthy ones [6]. However, the GLV-1h255 mediated overexpression of MMP-9 did not further enlarge these lymph nodes, as they had a similar size compared to GLV-1h68-treated animals and were significantly smaller than those in PBS treated animals. In accordance, Lavilla-Alonso et al. reported no increased tumor invasiveness by macrophage metalloelastase, another ECM degrading enzyme, when administered in combination with an oncolytic virus [35].

As MMP-9 can also induce angiogenesis by increasing the bioavailability of VEGF [29] which may stimulate primary tumor growth, we quantified the BVD in 
sections of tumors from infected and uninfected mice. The infected areas of both GLV-1h68- and GLV-1h255 treated tumors revealed similar BVD, which was significantly reduced compared to PBS-treated tumors.

Taken together, neither an increase in size of renal and lumbar lymph nodes, nor an induction of angiogenesis was observed in PC-3 tumor-bearing mice injected with GLV-1h255. Therefore, the final biological function of MMP-9 may strongly depend on the context and the local microenvironment of MMP-9 over-expression.

In this study, we propose that the enhanced oncolytic effect of GLV-1h255 could be due to the MMP9-mediated collagen IV degradation in PC-3 tumors.

Interestingly, the collagen IV content was significantly increased in GLV-1h68 infected tumors compared to PBS-treated tumors, which might be a result of an inflammatory reaction caused by viral infection. It has already been reported that tumor-targeted microbes, such as bacteria and viruses [27,36], induce an overwhelming inflammatory reaction in the tumor microenvironment, which is accompanied by an uncontrolled collagen deposition. In normal wounded tissue, inflammation is tightly controlled and ultimately leads to scar formation and healing. Tumors however, which also have been described by Dvorak as "wounds that do not heal" [37], do not provide an orderly environment for the resolution of an infection focus [28]. This may explain the excessive deposition of collagen IV in the context of infection. The significantly decreased collagen IV content in GLV-1h255 infected areas may be the reason for the significantly higher virus-titer in GLV-1h255 infected tumors, compared to those infected with GLV1h68, due to an accelerated extracellular cell to cell spreading. This would be in line with the findings of Ganesh et al. and Kim et al., who reported an increased tumor infection by adenoviruses after the degradation of ECM proteins [16,17]. This may furthermore explain the accelerated regression of GLV-1h255 infected tumors, as higher titers could result in an increased oncolysis, which has been shown to be an important factor for the therapeutic efficacy of VACV [27].

Collectively, our study revealed that the degradation of ECM within the tumor microenvironment can boost the oncolytic effect of rVACV. Moreover, we showed here that high levels of local, intra-tumoral ECM-degrading enzymes can be produced by virus-infected tumor cells themselves, certainly limiting systemic side effects.

\section{Conclusions}

In summary, the present study revealed that the degradation of collagen IV (ECM) by VACV-encoded MMP-9 may represent a new option to significantly enhance the oncolytic effect of rVACV in PC-3 xenografts. We confirmed that the degradation of collagen
IV facilitated viral infection of the tumor tissue, represented by significantly higher viral tumor titers and an accelerated tumor regression. Furthermore, both oncolytic viruses, parental GLV-1h68 and mmp-9-encoding GLV-1h255, significantly reduced the size of lumbar and renal lymph node metastases, indicating that MMP-9 enhances both virotherapy of the primary tumor and sustains the rVACV-metastasis reducing effect.

\section{Competing interests}

This work was supported by grants from Genelux Corporation (R\&D facility in San Diego, CA, USA). SW and UD received a postdoctoral fellowship, SS received a graduate fellowship awarded to the University of Würzburg, Germany by Genelux Corporation. QZ, RJA, NGC and AAS are employees of Genelux Corporation and have financial interests in Genelux Corporation.

\section{Authors' contributions}

SS conceived and designed the study, performed experiments, analyzed data and wrote the manuscript. SW helped to analyze tumor sections and helped to draft the paper. UD helped to analyze lymph nodes. UD also performed the in vitro virus replication assay. QZ, RJA and NGC constructed the recombinant vaccinia virus. AAS conceived the study and participated in coordination and drafting of the manuscript. All authors read and approved the final manuscript.

\section{Acknowledgements}

The authors thank J. Langbein, M. Jing and T. Trevino for excellent technical assistance, and J. Kramer for editorial support.

\section{Author details}

${ }^{1}$ Department of Biochemistry, Biocenter, University of Würzburg, Würzburg 97074, Germany. ${ }^{2}$ Genelux Corporation, San Diego Science Center, San

Diego, CA 92109, USA. ${ }^{3}$ Department of Radiation Oncology, Rebecca \& John Moores Comprehensive Cancer Center, University of California San Diego, La Jolla, CA 92093, USA

Received: 16 January 2012 Accepted: 20 August 2012

Published: 23 August 2012

\section{References}

1. Siegel R, Ward E, Brawley O, Jemal A: Cancer statistics, 2011: the impact of eliminating socioeconomic and racial disparities on premature cancer deaths. CA Cancer J Clin 2011, 61(4):212-236.

2. Cheng L, Zincke $H$, Blute ML, Bergstralh EJ, Scherer B, Bostwick DG: Risk of prostate carcinoma death in patients with lymph node metastasis. Cancer 2001, 91(1):66-73.

3. Mellor HR, Callaghan R: Resistance to chemotherapy in cancer: a complex and integrated cellular response. Pharmacology 2008, 81(4):275-300.

4. Bao S, Wu Q, McLendon RE, Hao Y, Shi Q, Hjelmeland AB, Dewhirst MW, Bigner DD, Rich JN: Glioma stem cells promote radioresistance by preferential activation of the DNA damage response. Nature 2006, 444(7120):756-760

5. Gnerlich J, Jeffe DB, Deshpande AD, Beers C, Zander C, Margenthaler JA Surgical removal of the primary tumor increases overall survival in patients with metastatic breast cancer: analysis of the 1988-2003 SEER data. Ann Surg Oncol 2007, 14(8):2187-2194.

6. Gentschev I, Donat U, Hofmann E, Weibel S, Adelfinger M, Raab V, Heisig M, Chen N, Yu YA, Stritzker J, et al: Regression of human prostate tumors and metastases in nude mice following treatment with the recombinant oncolytic vaccinia virus GLV-1h68. J Biomed Biotechnol 2010, 2010:489759.

7. Yu YA, Galanis C, Woo Y, Chen N, Zhang Q, Fong Y, Szalay AA: Regression of human pancreatic tumor xenografts in mice after a single systemic injection of recombinant vaccinia virus GLV-1h68. Mol Cancer Ther 2009, 8(1):141-151.

8. Yu Z, Li S, Brader P, Chen N, Yu YA, Zhang Q, Szalay AA, Fong Y, Wong RJ: Oncolytic vaccinia therapy of squamous cell carcinoma. Mol Cancer 2009, $8: 45$. 
9. Seubert CM, Stritzker J, Hess M, Donat U, Sturm JB, Chen N, von Hof JM, Krewer B, Tietze LF, Gentschev I, et al: Enhanced tumor therapy using vaccinia virus strain GLV-1h68 in combination with a beta-galactosidaseactivatable prodrug seco-analog of duocarmycin SA. Cancer Gene Ther 2011, 18(1):42-52.

10. Zhang Q, Yu YA, Wang E, Chen N, Danner RL, Munson PJ, Marincola FM, Szalay AA: Eradication of solid human breast tumors in nude mice with an intravenously injected light-emitting oncolytic vaccinia virus. Cancer Res 2007, 67(20):10038-10046.

11. McFadden G: Poxvirus tropism. Nat Rev Microbiol 2005, 3(3):201-213.

12. Smith $G L$, Moss $B$ : Infectious poxvirus vectors have capacity for at least 25000 base pairs of foreign DNA. Gene 1983, 25(1):21-28.

13. Frentzen A, Yu YA, Chen N, Zhang Q, Weibel S, Raab V, Szalay AA: AntiVEGF single-chain antibody GLAF-1 encoded by oncolytic vaccinia virus significantly enhances antitumor therapy. Proc Natl Acad Sci USA 2009, 106(31):12915-12920.

14. Haddad D, Chen NG, Zhang Q, Chen CH, Yu YA, Gonzalez L, Carpenter SG, Carson J, Au J, Mittra A, et al: Insertion of the human sodium iodide symporter to facilitate deep tissue imaging does not alter oncolytic or replication capability of a novel vaccinia virus. J Trans/ Med 2011, 9:36.

15. Brader P, Kelly KJ, Chen N, Yu YA, Zhang Q, Zanzonico P, Burnazi EM, Ghan RE, Serganova I, Hricak H, et al: Imaging a Genetically Engineered Oncolytic Vaccinia Virus (GLV-1 h99) Using a Human Norepinephrine Transporter Reporter Gene. Clin Cancer Res 2009, 15(11):3791-3801.

16. Ganesh S, Gonzalez Edick M, Idamakanti N, Abramova M, Vanroey M, Robinson M, Yun CO, Jooss K: Relaxin-expressing, fiber chimeric oncolytic adenovirus prolongs survival of tumor-bearing mice. Cancer Res 2007 , 67(9):4399-4407.

17. Kim JH, Lee YS, Kim H, Huang JH, Yoon AR, Yun CO: Relaxin expression from tumor-targeting adenoviruses and its intratumoral spread, apoptosis induction, and efficacy. J Natl Cancer Inst 2006, 98(20):1482-1493.

18. Nagase H, Woessner JF Jr: Matrix metalloproteinases. J Biol Chem 1999, 274(31):21491-21494.

19. Egeblad M, Werb Z: New functions for the matrix metalloproteinases in cancer progression. Nat Rev Cancer 2002, 2(3):161-174.

20. Page-McCaw A, Ewald AJ, Werb Z: Matrix metalloproteinases and the regulation of tissue remodelling. Nat Rev Mol Cell Biol 2007, 8(3):221-233.

21. Deryugina El, Quigley JP: Matrix metalloproteinases and tumor metastasis. Cancer Metastasis Rev 2006, 25(1):9-34.

22. Kessenbrock K, Plaks V, Werb Z: Matrix metalloproteinases: regulators of the tumor microenvironment. Cell 2010, 141(1):52-67.

23. Hamano $Y$, Zeisberg M, Sugimoto H, Lively JC, Maeshima $Y$, Yang C, Hynes RO, Werb Z, Sudhakar A, Kalluri R: Physiological levels of tumstatin, a fragment of collagen IV alpha3 chain, are generated by MMP-9 proteolysis and suppress angiogenesis via alphaV beta3 integrin. Cancer Cell 2003, 3(6):589-601

24. Heljasvaara R, Nyberg P, Luostarinen J, Parikka M, Heikkila P, Rehn M, Sorsa T, Salo T, Pihlajaniemi T: Generation of biologically active endostatin fragments from human collagen XVIII by distinct matrix metalloproteases. Exp Cell Res 2005, 307(2):292-304.

25. Ribatti D: Endogenous inhibitors of angiogenesis: a historical review. Leuk Res 2009, 33(5):638-644.

26. MMP-9 (matrix metallopeptidase 9 (gelatinase B, $92 \mathrm{kDa}$ type IV collagenase). http://AtlasGeneticsOncology.org/Genes/MMP9ID41408ch20q11.html.

27. Weibel S, Raab V, Yu YA, Worschech A, Wang E, Marincola FM, Szalay AA Viral-mediated oncolysis is the most critical factor in the late-phase of the tumor regression process upon vaccinia virus infection. BMC Cancer 2011, 11:68.

28. Schafer M, Werner S: Cancer as an overhealing wound: an old hypothesis revisited. Nat Rev Mol Cell Biol 2008, 9(8):628-638.

29. Hawinkels L, Zuidwijk K, Verspaget HW, de Jonge-Muller ES, van Duijn W, Ferreira V, Fontijn RD, David G, Hommes DW, Lamers CB, et al: VEGF release by MMP-9 mediated heparan sulphate cleavage induces colorectal cancer angiogenesis. Eur J Cancer 2008, 44(13):1904-1913.

30. Yang M, Jiang P, Yamamoto N, Li L, Geller J, Moossa AR, Hoffman RM: Real-time whole-body imaging of an orthotopic metastatic prostate cancer model expressing red fluorescent protein. Prostate 2005 62(4):374-379.

31. Apple JS, Paulson DF, Baber C, Putman CE: Advanced prostatic carcinoma: pulmonary manifestations. Radiology 1985, 154(3):601-604.
32. Wynder EL, Mabuchi K, Whitmore WF Jr: Epidemiology of cancer of the prostate. Cancer 1971, 28(2):344-360.

33. Kuriyama N, Kuriyama H, Julin CM, Lamborn K, Israel MA: Pretreatment with protease is a useful experimental strategy for enhancing adenovirusmediated cancer gene therapy. Hum Gene Ther 2000, 11(16):2219-2230.

34. Parato KA, Senger D, Forsyth PA, Bell JC: Recent progress in the battle between oncolytic viruses and tumours. Nat Rev Cancer 2005, 5(12):965-976.

35. Lavilla-Alonso S, Bauer MM, Abo-Ramadan U, Ristimaki A, Halavaara J, Desmond RA, Wang D, Escutenaire S, Ahtiainen L, Saksela K, et al: Macrophage metalloelastase (MME) as adjuvant for intra-tumoral injection of oncolytic adenovirus and its influence on metastases development. Cancer Gene Ther 2012, 19(2):126-134

36. Weibel S, Stritzker J, Eck M, Goebel W, Szalay AA: Colonization of experimental murine breast tumours by Escherichia coli K-12 significantly alters the tumour microenvironment. Cell Microbio/ 2008, 10(6):1235-1248

37. Dvorak HF: Tumors: wounds that do not heal. Similarities between tumor stroma generation and wound healing. N Engl J Med 1986, 315(26):1650-1659.

doi:10.1186/1471-2407-12-366

Cite this article as: Schäfer et al:: Vaccinia virus-mediated intra-tumora expression of matrix metalloproteinase 9 enhances oncolysis of PC-3 xenograft tumors. BMC Cancer 2012 12:366

\section{Submit your next manuscript to BioMed Central and take full advantage of:}

- Convenient online submission

- Thorough peer review

- No space constraints or color figure charges

- Immediate publication on acceptance

- Inclusion in PubMed, CAS, Scopus and Google Scholar

- Research which is freely available for redistribution 